\title{
Feasibility assessment of the interactive use of a Monte Carlo algorithm in treatment planning for intraoperative electron radiation therapy
}

\author{
Pedro Guerra , José M Udías , Elena Herranz , \\ Juan Antonio Santos-Miranda , Joaquín L Herraiz , \\ Manlio F Valdivieso , Raúl Rodríguez , Juan A Calama , \\ Javier Pascau' ,Felipe A Calvo , Carlos Illana , \\ María J Ledesma-Carbayo and Andrés Santos
}

\begin{abstract}
This work analysed the feasibility of using a fast, customized Monte Carlo (MC) method to perform accurate computation of dose distributions during pre- and intraplanning of intraoperative electron radiation therapy (IOERT) procedures. The MC method that was implemented, which has been integrated into a specific innovative simulation and planning tool, is able to simulate the fate of thousands of particles per second, and it was
\end{abstract}


the aim of this work to determine the level of interactivity that could be achieved. The planning workflow enabled calibration of the imaging and treatment equipment, as well as manipulation of the surgical frame and insertion of the protection shields around the organs at risk and other beam modifiers. In this way, the multidisciplinary team involved in IOERT has all the tools necessary to perform complex MC dosage simulations adapted to their equipment in an efficient and transparent way. To assess the accuracy and reliability of this $\mathrm{MC}$ technique, dose distributions for a monoenergetic source were compared with those obtained using a general-purpose software package used widely in medical physics applications. Once accuracy of the underlying simulator was confirmed, a clinical accelerator was modelled and experimental measurements in water were conducted. A comparison was made with the output from the simulator to identify the conditions under which accurate dose estimations could be obtained in less than $3 \mathrm{~min}$, which is the threshold imposed to allow for interactive use of the tool in treatment planning. Finally, a clinically relevant scenario, namely earlystage breast cancer treatment, was simulated with pre- and intraoperative volumes to verify that it was feasible to use the MC tool intraoperatively and to adjust dose delivery based on the simulation output, without compromising accuracy. The workflow provided a satisfactory model of the treatment head and the imaging system, enabling proper configuration of the treatment planning system and providing good accuracy in the dosage simulation.

Keywords: intraoperative electron beam radiation therapy, intraoperative radiotherapy, Monte Carlo treatment planning

(Some figures may appear in colour only in the online journal)

\section{Introduction}

Intraoperative electron radiation therapy (IOERT) refers to the delivery during surgery of a high dose of radiation, in a single session, directly to the post-resected tumour bed or to an unresected tumour. The dose to be delivered is usually prescribed in combination with external radiotherapy and/or chemotherapy, although it can occasionally be prescribed without any additional irradiation (Gunderson et al 2011).

Radiotherapy planning in IOERT is a complex task that requires the consensus of a multidisciplinary team to manage a given case. In this context, new tools have been proposed (Pascau et al 2012) for the study of different treatment alternatives during preplanning before surgery. Moreover, the ultimate decisions about cone dimensions or protection positioning are made during the final procedure, depending on the surgical findings: e.g. tumour size, resection margins, etc (Lamanna et al 2012). These last-minute modifications might require re-evaluation of the dose during surgery for quality control prior to dose delivery, a process that we will refer to as intraplanning.

In IOERT, Monte Carlo (MC) techniques have been mostly used to analyse the dosimetric characteristics of electron beams generated by the accelerator (Iaccarino et al 2011, Righi et al 2013) and to measure the influence of protection on dose delivery in phantom studies (Russo et al 2012). Nevertheless, the actual irradiation conditions in the operating room (OR) may differ significantly from those considered during simulations or phantom tests. Therefore, 
there are compelling reasons to include aspects such as irregularities of the surface being treated or the accumulation of biological fluids in the estimation of dose homogeneity and distribution (Rosi and Viti 2004). The translation of previous research into clinical practice has been limited because of the complexity and time-consuming numerical computations typically associated with MC methods.

To plan the intervention with the aid of a treatment planning system (TPS), the radiooncologist (RO), in co-operation with the surgeon, should allow the definition of plausible scenarios that could be found during the OR and simulate alternative treatments to define the best trade-off in each situation. Alternatively, the $\mathrm{RO}$ might be required to take into account the actual conditions in the OR and to recompute during surgery the dose distribution, before the dose is actually delivered. This is possible with the aid of an intraoperative CT and a tracking system (García-Vázquez et al 2013). In practice, to be useful in a clinical environment, the tool has to be accurate while allowing for a certain level of interactivity in performing both pre- and intraoperative dose computations, and this requirement imposes hard constraints on the computation time.

Despite its potential value, treatment planning in the context of IOERT has not been possible until recently, with the development of a specific TPS (radiance, GMV SA, Tres Cantos, Spain). It is believed that the lack of such a tool has limited the spread and acceptance of IOERT (Palta et al 1995). The TPS front-end allows region contouring, definition of the surgical frame and the representation of isodoses with the corresponding dose-volume histograms, based on the scenario being considered for dose delivery (Pascau et al 2012).

The ultimate goal of the framework around the TPS is to provide a flexible environment that enables accurate dose estimates within a period of a few minutes for any given CT volume of a patient, either pre- or intraoperatively, and for a given LINAC, either conventional or mobile.

This work analyses the capability of the IOERT TPS back-end for fast predictions of dose distributions during the clinical routine using optimized MC techniques, including irregularities and protection systems in the irradiation field. These capabilities are illustrated with two early breast cancer case studies, one showing application of the tool with preoperative images and the other with intraoperative images. Dose distributions were simulated by considering a standard configuration for this treatment: $5 \mathrm{~cm}$ applicator and $6 \mathrm{MeV}$ electron beam nominal energy.

\section{Material and methods}

The simulation framework, whose workflow is shown in figure 1, provided the means to calibrate the imaging equipment, model the treatment unit, including the applicator, and simulate any situation that could be found in the OR, while hiding the complexity of the set-up to the end user. This allowed the users to concentrate on the decisions required for proper treatment: beam energy, applicator configuration and protection positioning. The phase space (PHSP) was generated using experimental measurements with phantoms, as described later. A CT phantom was imaged to calibrate the imaging equipment and thus enable the transformation of Hounsfield numbers into tissue properties. With the aid of the TPS, the user could insert objects not present in the original CT to account for the actual settings of the treatment plan. Using all of this information, the MC module computed the delivered dose.

These calibration steps have been validated with the clinical set-up available at Clinica la Luz (Madrid, Spain), consisting of an Mx8000 IDT 16 CT Scanner (Koninklijke Philips 


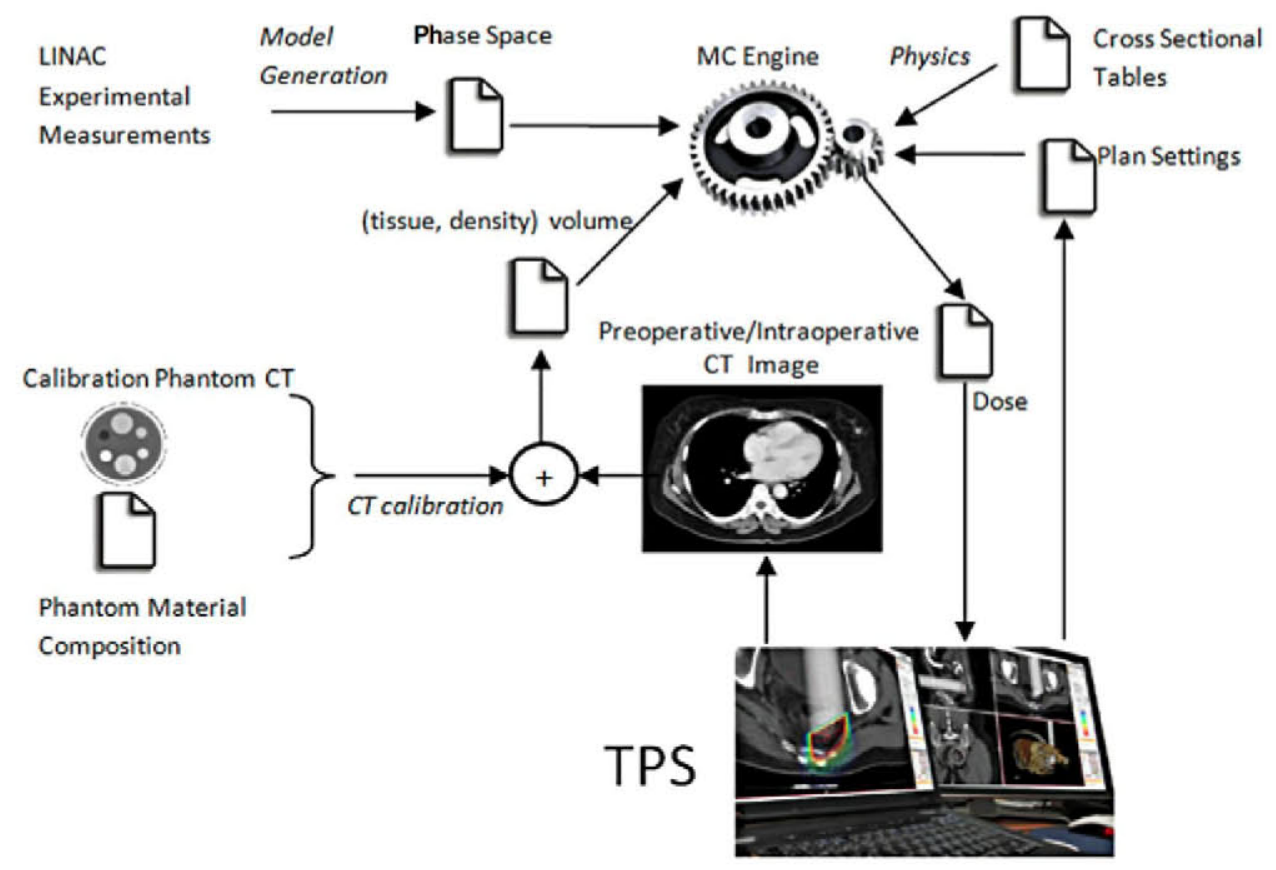

Figure 1. Technical workflow including configuration and user execution steps of the $\mathrm{MC}$ dose estimator included in the TPS system.

Electronics N.V., Amsterdam, The Netherlands) and a Varian 21EX linear accelerator (Varian Medical Systems, Palo Alto, CA, USA).

\subsection{Treatment unit simulation}

The physical properties of the electron beam for each energy and applicator diameter are characterized in the form of a PHSP model defined at a plane normal to the geometrical axis of the applicator. The PHSP describes the properties of the beam as a combination of multiple annular electron sources with nominal energy $E_{i}$ and radius $R_{i}$, radiating along a line defined by the PHSP plane at the angles $\left(\theta_{i}, \varphi_{i}\right)$ (Herranz 2013). A weight factor $w_{i}$ established the contribution of each elemental source to the beam. The model for the treatment unit, i.e. the computation of the individual weights $w_{i}$, was generated using a robust iterative maximum likelihood expectation maximization method (Herranz et al 2013), which provided a plausible set of weights for the existing experimental measurements. The iterative procedure consisted of two main steps: forward and backward projection. The forward projection provides, by the principle of linear superposition of doses, the dose $D^{\text {model }}$ produced by the current PHSP as a weighted linear combination of the individual dose footprints $d_{\mathrm{i}}$ generated by each elemental source. The measured dose $D^{\exp }$ was then compared with $D^{\text {model }}$, and correction factors were obtained for each voxel of the volume being considered. The correction factor for each bin is computed based on the ratio of measured to modelled dose.

To efficiently generate particles out of the PHSP during simulation, elemental sources were sorted and stratified based on the value of their individual weight factors. The $n_{1}$ microsources with the highest weight factor values $w_{i}$ belong to the first cluster, and similarly for the other 
microsources. The number of microsources $n_{j}$ in the cluster $C_{j}$ was selected so that it had a predefined a priori probability $P j$.

$$
\begin{aligned}
C_{j} & =\left\{w_{s_{j}} \ldots w_{s_{j}+n_{j}}\right\}, \forall(i<k): w_{i} \geq w_{k} \\
P_{j} & =\sum_{i=s_{j}}^{s_{j_{+}+1}-1} w_{i}
\end{aligned}
$$

The actual source $k$ was selected in two steps. Firstly, a random uniform number was generated to select the cluster $j$, and then a random element $k$ was taken from the subset $C_{j}$ using the rejection function $r(k)$.

$$
\begin{aligned}
& r(k)=\frac{w_{k}}{\max \left\{w_{k}\right\}}>\eta, w_{k} \in C_{j} \\
& \eta:: U(0,1)
\end{aligned}
$$

Particle variables $(E, \theta, \varphi)$ were generated randomly based on the nominal value of the randomly selected microsource $k$ and the discretization binning $(\Delta E, \Delta \theta, \Delta \varphi)$. Particle position $(x, y)$ in the PHSP plane was sampled directly from a ring with nominal radius $R_{k}$ using the following expressions:

$$
\begin{aligned}
& \xi:: U(0,1), \varsigma:: U(0,1) \\
& r_{2}=R_{k}+\frac{\Delta R}{2}, r_{1}=R_{k}-\frac{\Delta R}{2} \\
& \left.\begin{array}{l}
\theta=2 \pi \zeta \\
\rho=\sqrt{r_{1} \cdot r_{1} \cdot(1-\xi)+r_{2} \cdot r_{2} \cdot \xi}
\end{array}\right\} \begin{array}{l}
x=\rho \cdot \cos (\theta) \\
y=\rho \cdot \sin (\theta)
\end{array}
\end{aligned}
$$

The PHSP was located above the exit plane of the applicator in a plane normal to its geometrical axis. The applicator was inserted in the volume as a methacrylate hollow cylinder, as shown in figure 2 , overriding any material information coming from the CT image. With this approach, the effect of the bevel on the dose became part of the simulation, reducing the number of PHSP models to a single plane per nominal energy level and applicator diameter.

\subsection{Imaging equipment calibration}

Each voxel of the simulation volume was automatically assigned a tissue type and a density using a stoichiometric method (Schneider et al 1996, Vanderstraeten et al 2007) that generated device-specific conversion tables with the aid of a calibration phantom. In our implementation, model fitting was achieved by a constrained nonlinear multivariable optimization that searched for the set of cross-section values $\left(K_{\mathrm{ph}}, K_{\mathrm{coh}}, K_{\mathrm{KN}}\right)$ that minimized the maximum error between the model and the observed Hounsfield values in the calibration phantom. Constraints were imposed to avoid the convergence of cross-section fitting constants into negative values observed in the original algorithm (Kock and Schreuder 1996). Non-constrained results may provide a good fit but have no physical meaning.

A calibration phantom was acquired with an Mx8000 CT Scanner to validate the model-fitting algorithm developed for the scanner calibration. For this scanner, the algorithm provided the fitting constants: $K_{\mathrm{ph}} / K_{\mathrm{KN}}=1.001 \times 10^{-8}$ and $K_{\mathrm{coh}} / K_{\mathrm{KN}}=1.212 \times 10^{-5}$. These values provided the relationship between simulation tissues and Hounsfield values for this particular machine. 


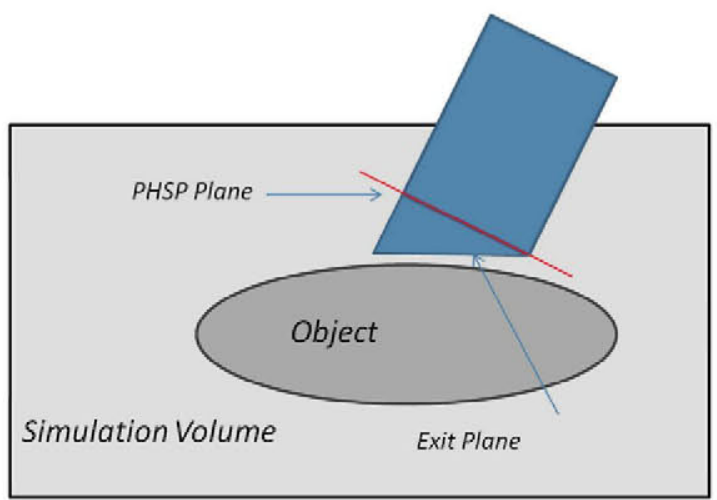

Figure 2. Diagram for the simulation of bevelled applicators.

\subsection{Protection and bolus insertion}

ROs need to define the expected surgical frame on the TPS by removing the tissues that will be resected or displaced during surgery from the simulation volume, as well as including new elements not found in the CT volume but present during dose delivery. Beam modifiers, such as bolus, are placed at the end of the applicator or on the patient surface, and shielding is used to protect critical structures (Beddar et al 2006). Twelve different protection materials, including lead, brass, aluminium and polymethyl methacrylate, among others, are currently available to define protection shields.

Protection systems and modifiers are 3D structures for which the user needs to specify dimension, orientation, shape and coordinates in the volume. During the planning procedure, these structures are inserted in the simulation volume, overriding the local tissue type and density given by the CT for each voxel and replacing it with the corresponding material type.

\subsection{MC dose estimation}

Dose to the medium was computed using a custom implementation of the dose planning method (DPM) (Sempau et al 2000), a 'mixed'class algorithm that runs in a fraction of the time of the original computer program. DPM uses an analogue simulation of photon interactions and a class II condensed history method (Kawrakow and Bielajew 1998) for electron simulation, treating large energy-transfer collisions in an analogue sense and using the continuous slowing-down approximation (CSDA) to model small energy-loss collisions. Cross-sections and mean free paths for each energy and material type were computed and tabulated offline. During simulation, these physical parameters were estimated by interpolation.

In our implementation, computational acceleration is achieved by optimizing the algorithm flow and efficiently using the hardware resources available in a modern multicore computer. The graphic processor unit, which can also be used to speed up the computation of dose (Jia et al 2011, Hissoiny and Ozell 2011), is devoted to image rendering in the TPS.

2.4.1. Parallelization. Particle transport is inherently a parallel problem and, therefore, porting the algorithm onto a multicore architecture was relatively straightforward. Execution is divided into threads, each simulating a subset of the total number of particles. To avoid any overhead due to synchronization, threads are loosely coupled. That is, just read-only data 
structures were shared, and there is a single common critical section to monitor conditions for stopping the simulation.

2.4.2. Algorithm. With respect to electrons, our implementation incorporates a dual random hinge approach (Bielajew and Wilderman 2000), in which energy loss and multiple elastic scattering are fully decoupled. Additionally, the management of secondary particles below cut-off values is changed in the simulator engine to prevent their insertion into the simulation queue, thus reducing the workload. Random numbers are generated using a re-entrant combination of linear generators (L'Ecuyer 1998), which are efficient to execute while being sufficiently random to pass demanding statistical tests (L'Ecuyer and Hellekalek 1998, L'Ecuyer and Panneton 2005). With respect to photons, Compton scattering is the main mechanism for dose delivery at the energies under consideration. Photoelectric absorption is relevant at low energies and for high atomic numbers. Pair production becomes significant at the high end of the energy range and for materials with high atomic numbers. Photon transport is implemented following the $\delta$-scattering method (Sempau et al 2000). In the standard approach, a sequence of Compton interactions ends with either a photoelectric interaction or a pair production. In our implementation, both interactions were forced, i.e. a fraction of the photon energy was absorbed as a photoelectric interaction and another fraction produces an electron/ positron pair. The fraction that undergoes each process depended on the relative probability of each interaction at the given photon energy.

2.4.3. Compiler. Iterative loops are reordered and unrolled to reduce the use of conditional branches, thus avoiding costly pipeline flushes. Pointers are de-aliased at function calls to provide the compiler with more freedom in code reordering and optimization.

2.4.4. Hardware. Particles are generated and simulated in blocks to increase data and code locality, and to improve throughput. The memory layout of interpolation spline coefficients is arranged to have them stored in consecutive memory positions, so that they are fetched from external memory in a single access and stored in a single cache line, thus reducing the number of memory accesses and cache misses.

The statistical uncertainty of the simulated dose is determined using the history-by-history method (Walters et al 2002), which provides the estimated uncertainty $\sigma_{j}^{2}$ for each voxel as:

$$
\sigma_{j}^{2}=\frac{1}{N-1}\left(\frac{\sum_{i=1}^{N} D_{i}^{2}}{N}-\left(\frac{\sum_{i=1}^{N} D_{i}}{N}\right)^{2}\right),
$$

where $N$ is the number of simulated histories and $D_{i}$ is the deposited dose per voxel. Periodically during the simulation, the maximum uncertainty is calculated for those voxels whose dose is higher than $50 \%$ of the maximum dose $\left(D_{\max }\right)$. Simulation execution ends when the maximum uncertainty is lower than a value specified by the user, which in our simulations was usually set to $2 \%$.

At the end of the simulation, the worst-case uncertainty $\sigma_{\max }^{2}$ is calculated for those voxels where the dose was higher than $50 \%$ of $D_{\max }$. Simulation efficiency $\varepsilon$, is defined as

$$
\varepsilon=\frac{1}{\sigma_{\max }^{2} T},
$$

where $T$ is the CPU time required to run the simulation, is reported after every simulation. 


\subsection{Dose verification}

The accuracy of the underlying algorithm to reproduce dose distributions, obtained either by using other MC codes (Sempau et al 2000) or measured experimentally (Chetty et al 2007), has already been shown by other authors. However, the proposed implementation introduces multiple optimizations and modifications that had to be verified.

The verification of dose distributions was performed in three steps. First, DOSRZnrc (Kawrakow and Rogers 2000), which is widely used by the medical physics community for the calculation of dose deposition, was used as a reference to assess the accuracy of the MC simulation. Second, experimental measurements in a water tank were compared with simulated dose profiles using a PHSP model of the accelerator obtained with our iterative method. Finally, monitor units (MU) required to deliver a given prescribed dose with different bevel angles and field sizes were computed following the standard protocol used in the clinic, and these values were compared with the dose profiles obtained using the $\mathrm{MC}$ method.

Dose distributions for a monochromatic parallel source, with beam energy between 4 and $20 \mathrm{MeV}$, in water and slab phantoms were simulated to compare our MC simulation with DOSRZnrc. Two alternative slab phantoms, consisting of water, aluminium and lung, were used to stress the code with a high-energy beam and a sharp inhomogeneity (Rogers and Mohan 2000).

PHSP models for the Varian 21EX system, with nominal energy in the 6-20 MeV range and an applicator diameter of $6 \mathrm{~cm}$, were generated using the iterative method described in section 2.1. For each energy, a PHSP model was estimated using water measurements with a right-angled applicator. The $6 \mathrm{~cm}$ applicator was used as an example to show the capability of reproducing dose distributions at different energies and bevel angles. The computation of dose footprints $d_{\text {i }}$ was carried out offline in a computer cluster, with a computational effort equivalent to 10 months of a single-core processor. Once the dose footprints were computed, the iterative algorithm found a set of weights fitting the estimated dose to the measured dose in water for this specific accelerator. A full iteration of the fitting algorithm was completed when the multiplicative factor had been computed for all bins, and all bins in the PHSP were updated using these factors. The entire fitting procedure typically required around 200 full iterations before convergence, which took a few minutes to fit each energy and cone diameter using a single-core processor.

MC simulations were then used to obtain the number of MUs required to deliver a given prescribed dose with different bevel angles and energies, and these values were compared with those obtained through measurement and hand computation (Cygler et al 1997).

Following the recommendations of the Spanish Medical Physics Society, absorbed dose to water protocols were used for calibration (IAEA2000). Relative measurements were taken in water and air with an MP3 radiation field analyser (PTW, Freiburg, Germany), equipped with a p-type electron diode detector (Scanditronix Medical AB, Uppsala, Sweden). Another diode detector was placed in the periphery of the radiation field during scanning to acquire reference conditions. The TANDEM electrometer (PTW) was used for all scanning measurements. The uncertainty of such a measurement protocol is less than $1 \%$ in dose and $0.5 \mathrm{~mm}$ in distance. $P$-type electron diodes were also used to measure cone output factors in water (Björk $e t$ al 2004), with an uncertainty lower than $0.5 \%$.

MUs were calibrated for each energy to provide $1 \mathrm{cGy}$ at the maximum of the percentage depth dose (PDD) in water, per MU, with the standard $10 \mathrm{~cm} \times 10 \mathrm{~cm}$ applicator and a source-to-surface distance (SSD) of $100 \mathrm{~cm}$. Output factors (OF) were computed (Almods 1999) at the maximum of the clinical axis with three different SSDs for each 


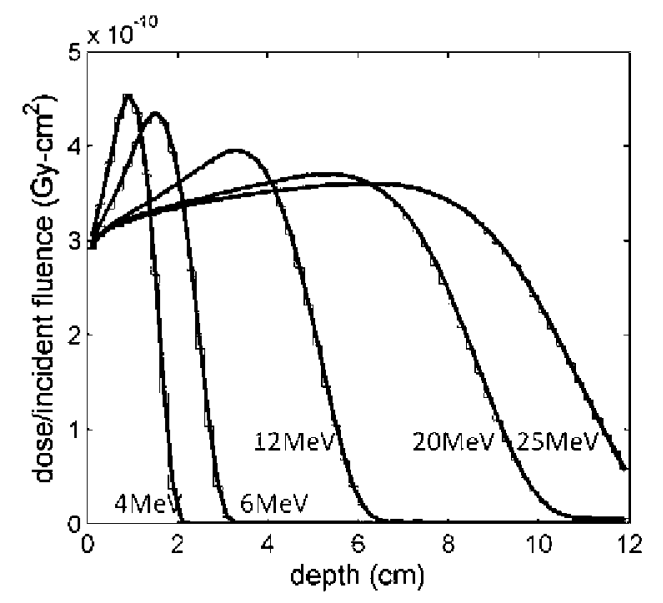

Figure 3. PDD results for a broad mono-energetic electron beam with nominal energies in the range of $4-25 \mathrm{MeV}$, computed with the implemented $\mathrm{MC}$ code (solid-black) and DOSZRnrc (dashed-blue).

bevel-diameter combination and intermediate distances were estimated by interpolation, taking a $10 \mathrm{~cm} \times 10 \mathrm{~cm}$ applicator as a reference. To estimate MU with the $\mathrm{MC}$ method, a gain factor was computed for each PHSP so that the simulation output was in the same units as the water measurements. In this way, MUs were estimated for a $127 \mathrm{~cm}$ applicator using the OFs computed previously.

\section{Validation and results}

All calculations reported here were performed on a PC running Microsoft 64-bit Windows 7 with an Intel $2.93 \mathrm{GHz}$ i7 processor and $8 \mathrm{~GB}$ RAM.

\subsection{Dose simulation with monochromatic sources}

Dose-depth profiles in a water phantom (figure 3) and slab phantoms (figures 4 and 5) were simulated using a monoenergetic beam at different energies. Dose profiles in water of figure 3 confirmed agreement between the reference (DOSRZnrc) and computed profiles, with a difference between curves lower than $1.5 \%$ of the maximum dose at max.

The slab phantom used in figure 4 demonstrate the accuracy of the MC model to reproduce dose profiles in the energy range of interest for procedures with protection around the lung, with a relative error below $1.5 \%$. Additionally, the slab phantom described in figure 5 was used to stress the code with a high-energy beam and a sharp inhomogeneity (Rogers and Mohan 2000). The results show good agreement between the profiles, with a slight mismatch along the lung. In fact, this mismatch was smaller than that found using the original computer program (Sempau et al 2000), as shown in the upper-right corner of the figure 5. The differences were attributed to the use of a finer grid for the precomputed cross-sections, as well as to the algorithmic changes introduced.

These results show that the MC method implemented accurately predicted dose profiles in the presence of sharp inhomogeneities, which is a common scenario in IOERT where high atomic number $(Z)$ materials are used to shield organs at risk. In addition, the 


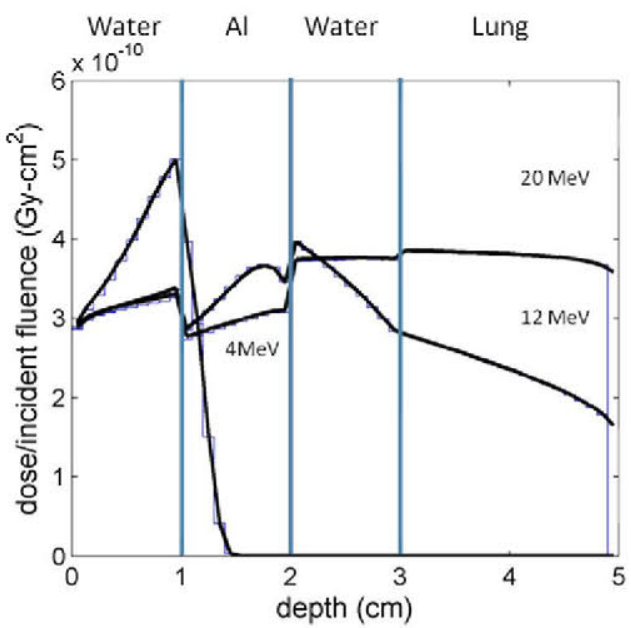

Figure 4. $\mathrm{PDD}$ in a $5 \mathrm{~cm}$ water/aluminium/water/lung the slab phantom for a broad mono-energetic electron beam with nominal energies in the range of $4-20 \mathrm{MeV}$, computed with the implemented MC code (solid-black) and DOSZRnrc (dashed-blue).

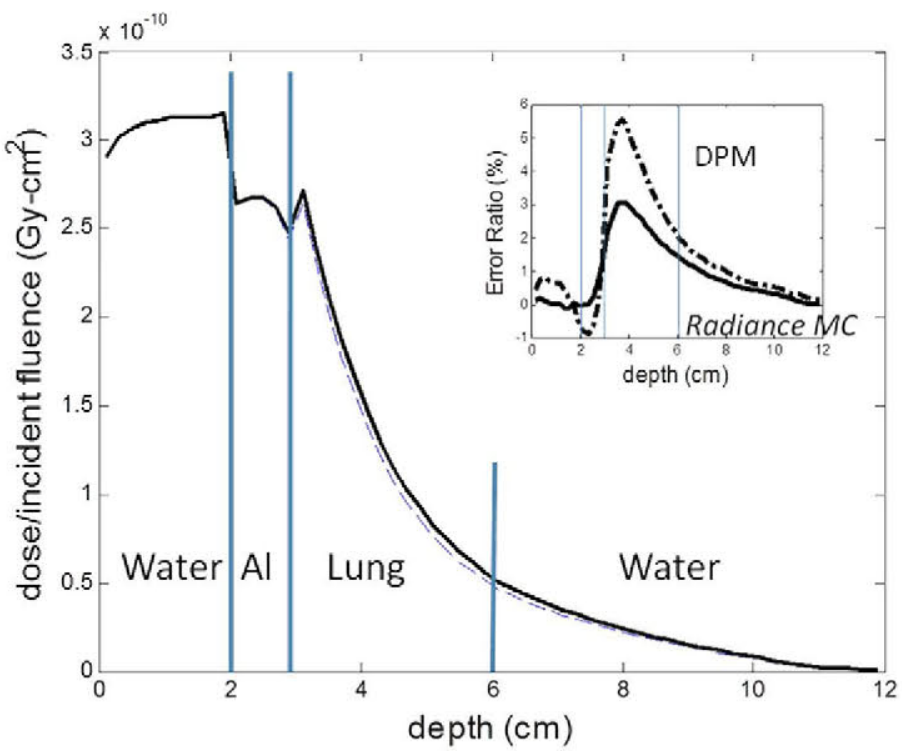

Figure 5. Comparison of depth-dose curve in a water/al/lung/water slab phantom for $1.5 \times 1.5 \mathrm{~cm}^{2} 20 \mathrm{MeV}$ mono-energetic electron beam, radiating from a uniform point source at $100 \mathrm{~cm}$ incident on the phantom, computed with the implemented MC code (solid black) and DOSRZnrc (dashed-blue). The relative error with respect to the reference is shown in the upper right corner for the MC code (solid black) and the original implementation of the algorithm (dashed black). Simulations are run with the cutoff energies $200 \mathrm{keV}$ and $50 \mathrm{keV}$ energy for electrons and photos, respectively. 


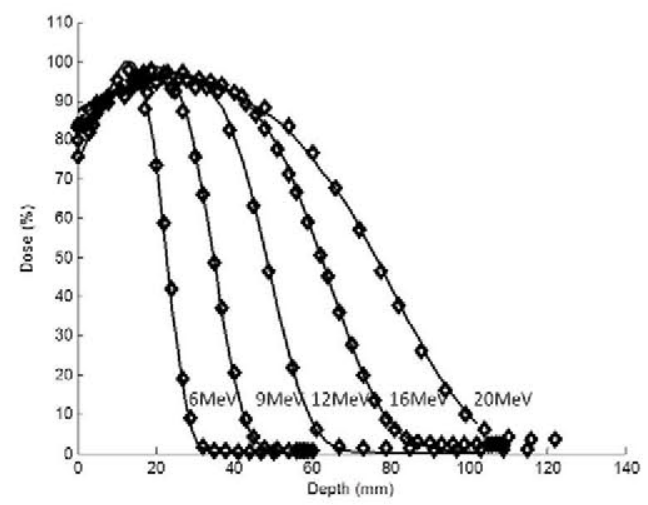

Figure 6. Simulated (solid) and measured (diamonds) PDD curve for a $6 \mathrm{~cm}$ applicator at nominal head energies of $6,9,12,16$, and $20 \mathrm{MeV}$.

computations required a fraction of the time needed by the original computer program, as discussed in section 3.3 .

\subsection{Treatment unit simulation results}

Figure 6 compares the experimental and simulated PDD curves for a $6 \mathrm{~cm}$ right-angled applicator for nominal energies between $6-20 \mathrm{MeV}$, while figure 7 compares the experimental and simulated lateral profiles at $16 \mathrm{MeV}$ for a $6 \mathrm{~cm}$ bevelled applicator, with bevel angles between $15^{\circ}$ and $45^{\circ}$. Figure 7 shows the ability to model the effect of the bevel and to predict the shape of the lateral dose profiles. The fact that measurements with bevelled applicators were not employed to fit the PHSP must be taken into account, and thus they are a good indicator of the validity of the PHSP $+\mathrm{MC}$ algorithm for this configuration. However, the ability to reproduce measurements depends greatly on the PHSP model, and, therefore, a complete validation of the iterative methods used to model the phase space is required; such a study falls beyond the scope of this work and has been addressed by (Herranz et al 2013).

The gamma function (Low et al 1998) was used to compare the experimental profiles with the simulation output for all energies and bevel angles, with the result that the gamma function was smaller than that corresponding to a dose difference of $3 \%$ and to estimating distance to an agreement of $3 \mathrm{~mm}$, which is well within the needs of IOERT.

As a further test of the suitability of the MC + PHSP for this case, MUs were measured and computed for dose distributions in a water phantom with an SSD of $127 \mathrm{~cm}$ and bevels of $0^{\circ}, 15^{\circ}, 30^{\circ}$ and $45^{\circ}$ to verify that the resulting values could be trusted. Table 1 summarizes the required number of MUs estimated by the MC tool for each energy and bevel, and enabled comparison of this value with manually computed MUs using the measurement data.

The ratio between values calculated by the $\mathrm{MC}$ tool and the measurements confirmed their consistency, with a mean value of 1.004 and a standard deviation of 0.013 for the $6 \mathrm{~cm}$ applicator. By averaging these values for all measured applicator diameters $(3,4,5,6,7,8$ and $9 \mathrm{~cm})$, the mean value of the ratio was 0.999 and the standard deviation was 0.016 , values that are comparable with those reported by other authors (Cygler et al 2004) for electron beams with a different MC-calculation engine. 

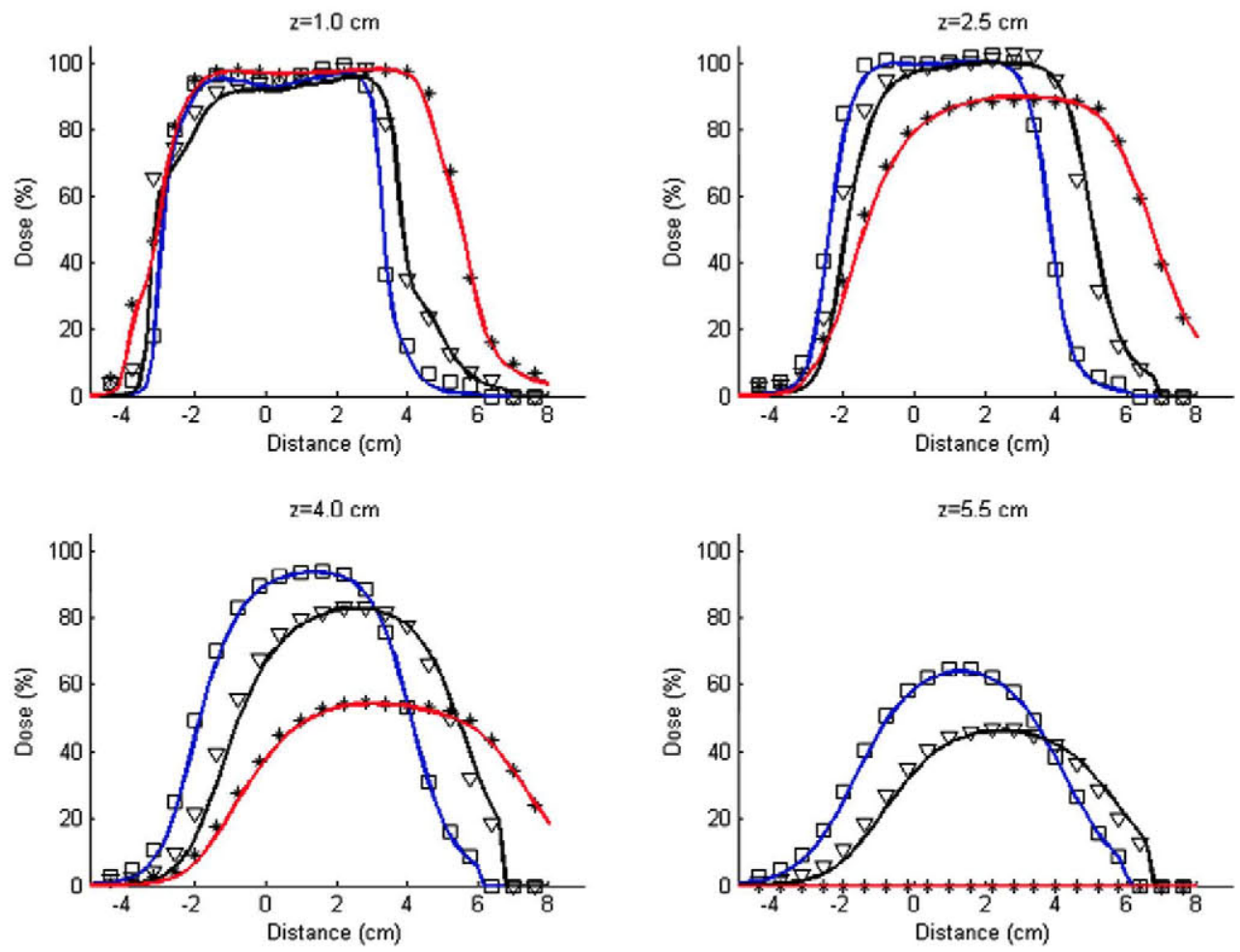

Figure 7. Simulated (solid line) and measured (scattered points) lateral dose for a $6 \mathrm{~cm}$ applicator at nominal head energy of $16 \mathrm{MeV}$ and bevels of $15^{\circ}$ (blue-square), $30^{\circ}$ (black-diamond) and $45^{\circ}$ (red-star) at depths equal to $1 \mathrm{~cm}, 2.5 \mathrm{~cm}, 4 \mathrm{~cm}$ and $5.5 \mathrm{~cm}$.

\subsection{Algorithm optimization and profiling}

The algorithm was profiled to identify and progressively optimize bottlenecks. Particular attention was paid to the generation of random numbers and the sampling of different probability density functions, portions of code used intensively in the MC.

The actual load distribution depends on the object being modelled, as well as the energy and applicator size. With the aim of reducing the computation time in worst-case scenarios, only configurations with wide applicators and high energy were considered for code profiling. The distribution of workload in a water phantom with an $8 \mathrm{~cm}$ applicator at $20 \mathrm{MeV}$, summarized in table 2 , is very similar to the distribution in the reference implementation of the algorithm (Sempau et al 2000). Simulation effort was divided into four main modules: particle generation, simulation management, particle transport and energy recording. The simulation of electron physics took most of the computational effort (71.7\%). The simulation of electrons was split into the implementation of the CSDA and the simulation of discrete events, such as the generation of bremsstrahlung photons. In both cases, the computational effort was divided into the actual transport of particles within the volume (geometry) and statistical sampling of the different physical processes to compute displacement and rotation angles.

The sampling of the PHSP was costly without stratification. Four layers were enough to reduce the effort of particle generation from $30 \%$ to $3.9 \%$ of the total simulation time. Moreover, random number generation, which was used at multiple points, accounting for 
Table 1. Comparison of MU Monte Carlo versus measurements for all nominal energies and bevels for an applicator diameter of $6 \mathrm{~cm}$, for a prescribed dose of $21 \mathrm{~Gy}$ at $d_{\max }$ along the beam central axis.

\begin{tabular}{|c|c|c|c|c|c|c|c|c|c|c|}
\hline \multirow[b]{2}{*}{ Bevel } & \multicolumn{2}{|c|}{$6 \mathrm{MeV}$} & \multicolumn{2}{|c|}{$9 \mathrm{MeV}$} & \multicolumn{2}{|c|}{$12 \mathrm{MeV}$} & \multicolumn{2}{|c|}{$16 \mathrm{MeV}$} & \multicolumn{2}{|c|}{$20 \mathrm{MeV}$} \\
\hline & $\mathrm{MC}$ & MC/Meas & $\mathrm{MC}$ & MC/Meas & $\mathrm{MC}$ & MC/Meas & $\mathrm{MC}$ & MC/Meas & $\mathrm{MC}$ & MC/Meas \\
\hline 0 & 4215 & 1.006 & 3018 & 0.995 & 2692 & 0.998 & 2596 & 0.998 & 2596 & 1.001 \\
\hline 15 & 4322 & 0.986 & 3118 & 0.997 & 2750 & 0.995 & 2612 & 0.995 & 2612 & 0.999 \\
\hline 30 & 4462 & 1.004 & 3164 & 1.010 & 2765 & 1.005 & 2607 & 1.010 & 2566 & 1.009 \\
\hline 45 & 4467 & 0.989 & 3110 & 1.001 & 2749 & 1.020 & 2595 & 1.026 & 2606 & 1.044 \\
\hline
\end{tabular}

Table 2. $\mathrm{MC}$ code profiling for an $8 \mathrm{~cm}$ applicator in a water phantom for a $20 \mathrm{MeV}$ source, expressed as percentage of the total simulation time.

\begin{tabular}{|c|c|c|c|c|}
\hline \multicolumn{4}{|l|}{ MC simulation step } & \multirow{2}{*}{$\begin{array}{c}\begin{array}{c}\text { Percentage } \\
\text { of total time }\end{array} \\
3.9 \%\end{array}$} \\
\hline Particle Generation & & & & \\
\hline \multirow{7}{*}{ Particle Matter Interactions } & \multirow{6}{*}{ Electrons $(71.7 \%)$} & & Geometry & $19.1 \%$ \\
\hline & & CSDA & Physics & $33 \%$ \\
\hline & & & Sampling & \\
\hline & & & Geometry & $3.4 \%$ \\
\hline & & Discrete & Physics & $16.2 \%$ \\
\hline & & & Sampling & \\
\hline & Photons $(1.8 \%)$ & & & $1.8 \%$ \\
\hline Energy Tallying & & & & $18.3 \%$ \\
\hline MC Simulation Manager & & & & $2.1 \%$ \\
\hline Other & & & & $2.2 \%$ \\
\hline
\end{tabular}

$12 \%$ of the computing time. This fact justifies the use of an efficient re-entrant combination of linear generators.

To assess the impact of the different modifications introduced to the original implementation, the efficiency gain provided by the MC tool in every run was evaluated for a fixed number of particles after algorithmic improvements (CODE OPT), memory layout and compiler considerations (MEM OPT) and simulation parallelization into multiple threads (THREAD). These values were compared with the efficiency of original code. The results, summarized in figure 8 , show that the efficiency doubled after algorithmic and memory optimizations, and an additional factor of four was achieved by execution on multicore architecture. Because the underlying physics were the same, the efficiency gains were due mostly to a reduction in the computation time.

The motivation behind these optimizations was the intention to use the TPS and the $\mathrm{MC}$ to perform treatment preplanning and intraplanning. The intraoperative simulation helps to take into consideration the actual setting in the operating room and to validate hypotheses assumed during planning before the actual delivery. In clinical practice, where the patient is anaesthetized and undergoing surgery, the aim is that the MC computation should not delay the procedure, and it is necessary that the simulation be completed in less than $3 \mathrm{~min}$. In dose simulations with a water phantom with $1 \times 1 \times 1 \mathrm{~mm}^{3}$ voxels, it was observed (figure 9) that such a restrictive time constraint was not met when wide applicators and high energies were used, even for a relatively lax uncertainty requirement of $4 \%$ of the dose at the maximum, which required the simulation of more than 30 million particles. 


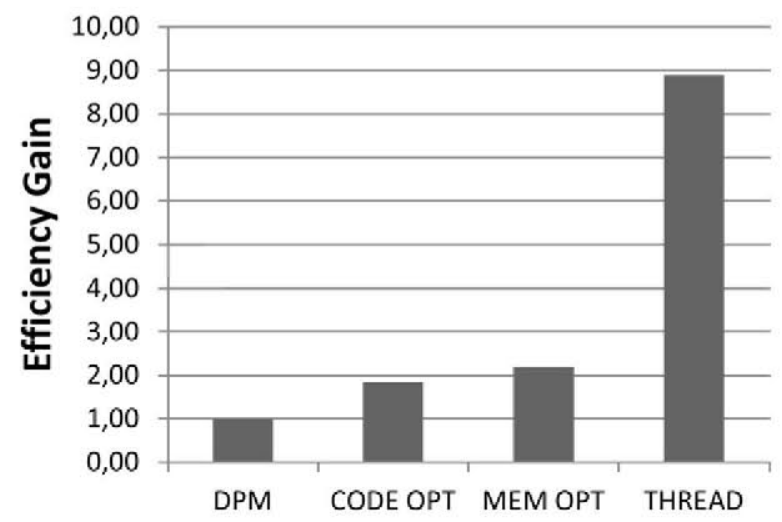

Figure 8. Efficiency gain for the original code running on 1 core (DPM), the optimized algorithm (CODE OPT) running on 1 core, the former algorithm with the new memory layout and compiler-oriented optimizations (MEM OPT) running on 1 core, and finally with execution divided into 6 threads (THREAD), running on 4 cores. Simulations are run in a $3 \times 3 \times 3 \mathrm{~mm}^{3}$ voxelized water phantom with a $20 \mathrm{MeV}$ monochromatic source.

In figure 9 it is observed that, if we restrict ourselves to the combinations used most frequently in clinics (where energies above $12 \mathrm{MeV}$ are rarely used) and consider a larger voxel size $\left(8 \mathrm{~mm}^{3}\right.$ instead of $\left.1 \mathrm{~mm}^{3}\right)$, accurate dose distributions, with uncertainties lower than $2 \%$, could be completed in less than 3 min in most scenarios.

\subsection{Dose simulations with the complete workflow}

Two representative scenarios of treatment planning in breast cancer are presented to illustrate the utility and limitations of the workflow that was developed and implemented. In case 1, the preoperative image would typically be used to analyse possible treatment approaches. In case 2, the intraoperative CT image would be used to confirm whether the actual set-up in the operating room, including the applicator and position of the protection, meets the prescribed requirements before dose delivery.

CT studies were acquired at Hospital Gregorio Marañón in Madrid on a Toshiba Aquilion LB scanner (Toshiba Medical Systems Corporation, Tochigi, Japan) with the following parameters: voltage $120 \mathrm{KVp}$, current $40 \mathrm{~mA}$, slice thickness $3 \mathrm{~mm}$ and pixel spacing $1.8 \times 1.8 \mathrm{~mm}^{2}$ for case 1 , and slice thickness $5 \mathrm{~mm}$ and pixel spacing $1 \times 1 \mathrm{~mm}^{2}$ for case 2 . The intraoperative CT was obtained as part of a research protocol in which a specifically designed couch was used to transport the patient from the OR to the CT scanner under anaesthesia, with the applicator treatment position fixed using an articulated arm. This protocol was designed to evaluate the difficulties and contributions of intraoperative CT imaging in IOERT scenarios. The optimal clinical protocol definition is still under investigation.

Case 1 emulates a preplanning workflow. In this scenario, the RO would use the preoperative image to segment the planning target volume (PTV), organs at risk and fluids on the tumour bed; select the treatment configuration (applicator position, diameter and bevel angle, as well as the nominal energy); and place the beam modifiers depending on several factors (size of the treatment target, anatomical structures, candidate areas to be protected). In this simulated scenario, shown in figure 10 , the RO would prescribe 21 Gy to irradiate the PTV around the tumour bed delineated as a truncated cone shape, with a base of $40 \mathrm{~mm}$ and a height of $15 \mathrm{~mm}$. The user, in this particular case an RO with IOERT experience following 

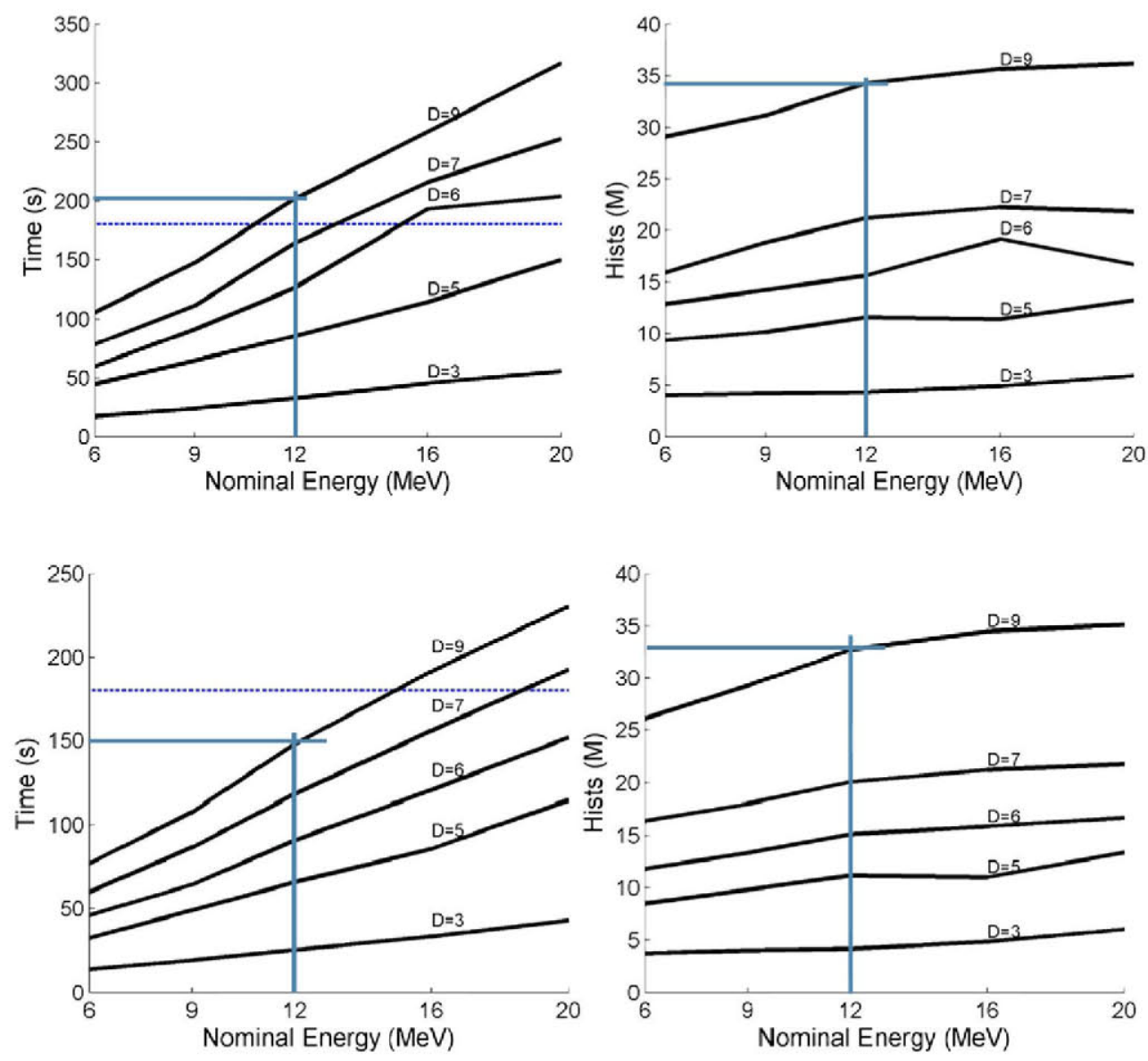

Figure 9. (Upper-left) Simulation time required to estimate dose with an uncertainty lower than $4 \%$ for a $161 \times 140 \times 161$ water phantom, with $1 \times 1 \times 1 \mathrm{~mm}^{3}$ per voxel and for applicator diameters in the range of $3 \mathrm{~cm}-9 \mathrm{~cm}$. (Upper-right) Number of simulated primary particles in millions. (Lower-left) Simulation time required to estimate dose with an uncertainty lower than $2 \%$ for the same phantom with a $2 \times 2 \times 2 \mathrm{~mm}^{3}$ voxelization and (lower-right) the required number of simulated primary particles.

the standard clinical protocol that he follows in the $\mathrm{OR}$, positioned a $5 \mathrm{~cm}$ diameter applicator and inserted an $8 \mathrm{~cm}$ diameter $/ 5 \mathrm{~mm}$ thick cylindrical shield $(3 \mathrm{~mm}$ aluminium $/ 2 \mathrm{~mm}$ brass $)$ at the location where he considered that the ribs and lung would be best protected. With these settings, the RO and the radiation physicist expected to protect the lungs and ribs completely, while the delineated target volume would receive at least $90 \%$ of the prescribed dose. Multiple simulations, with slight variations in the applicator, protection positions and angles, were run to identify the optimal applicator location in terms of PTV coverage. This case used a twolayered disc configuration, which is frequently utilized in practice; the top disc with the lower $Z$ slows down beam particles and absorbs backscattered particles (Catalano et al 2007), and the bottom disc with the higher $Z$ stops the residual electrons.

Dose was estimated by considering a $6 \mathrm{MeV}$ electron beam generated by a Varian 21EX linear accelerator. To obtain a statistical dose uncertainty $\sigma_{\max }{ }^{2}$ below $2 \%$, approximately 


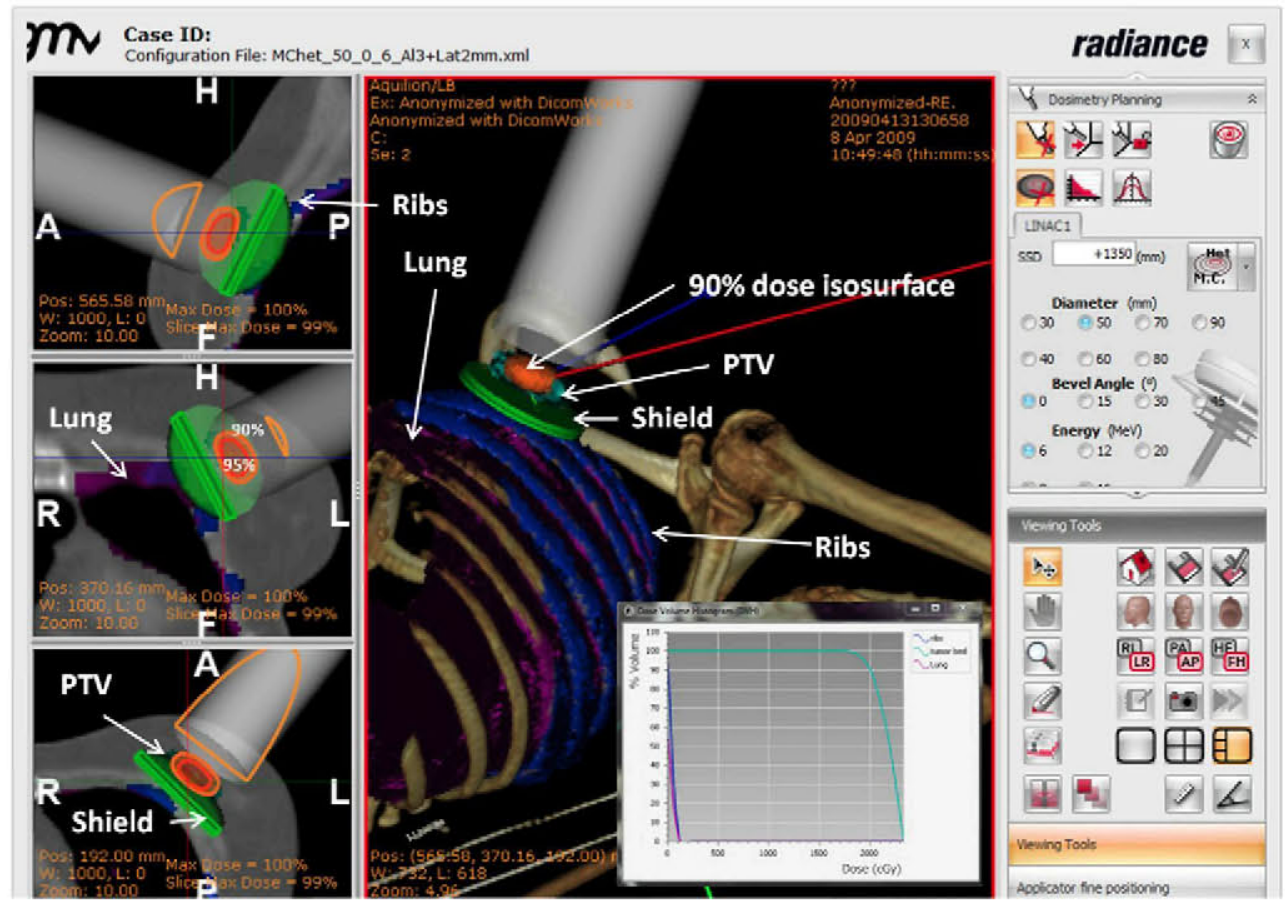

Figure 10. TPS interface showing the MC simulation output for a treatment planning with a $6 \mathrm{MeV}$ electron beam and a $5 \mathrm{~cm}$ applicator (case 1). The breast is protected with a composite shield that is positioned by a RO. The pixel size of the preoperative CT image is $1.8 \times 1.8 \times 3 \mathrm{~mm}^{3}$. Isodoses curves are represented for the $95 \%$ (red) and $90 \%$ (orange) of the maximum dose.

11.2 million particles, with an execution time of less than $35 \mathrm{~s}$, were simulated in each MC run. This execution time would allow for a certain degree of interactivity in the planning process.

After several iterations, the RO determined that in the best-case scenario (figure 11, top left) the PTV was not completely enclosed by the $90 \%$-isodose curve obtained with the $5 \mathrm{~cm}$ applicator, and it was estimated that, at best, $88 \%$ of the tumour volume was inside the $90 \%$-isodose curve. The same setting was simulated with a $6 \mathrm{~cm}$ applicator (figure 11 , top right), and in this case the delineated PTV was completely enclosed by the $90 \%$-isodose curve, allowing for a margin of safety in the OR for position of the applicator and/or protection misalignments with respect to the optimum location. These simulation results are in accordance with the new standard treatment proposed by the European Institute of Oncology where the recommended applicator size is now $6 \mathrm{~cm}$, with an occasional use of $5 \mathrm{~cm}$ for very small lesions (Dall'Oglio 2013).

In case 2, the applicator was placed in the TPS to reproduce the real applicator position, as displayed on the CT image (figure 12). The CT scan was obtained with the protection in place, although other clinical protocols for breast cancer treatment avoid the use of any protection (Fastner et al 2013). The reconstructed CT image showed severe artefacts due to the high density of the shielding materials. To simulate dose deposition, the applicator was positioned so that it overlapped the end of the applicator visible in the image, protection was placed on top of the protection with artefact visible on the image, and the tissue between the applicator and the protection was segmented as soft tissue. This workaround overcomes the limitations 


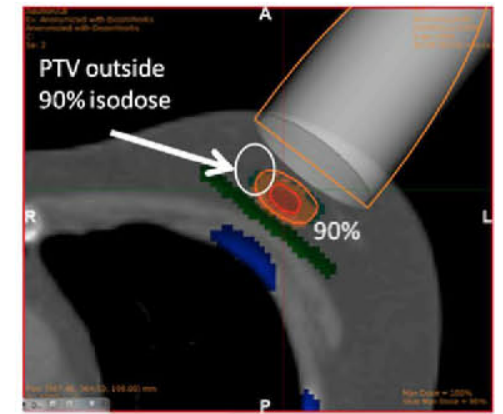

Dose Volume Histogram $5 \mathrm{~cm}$

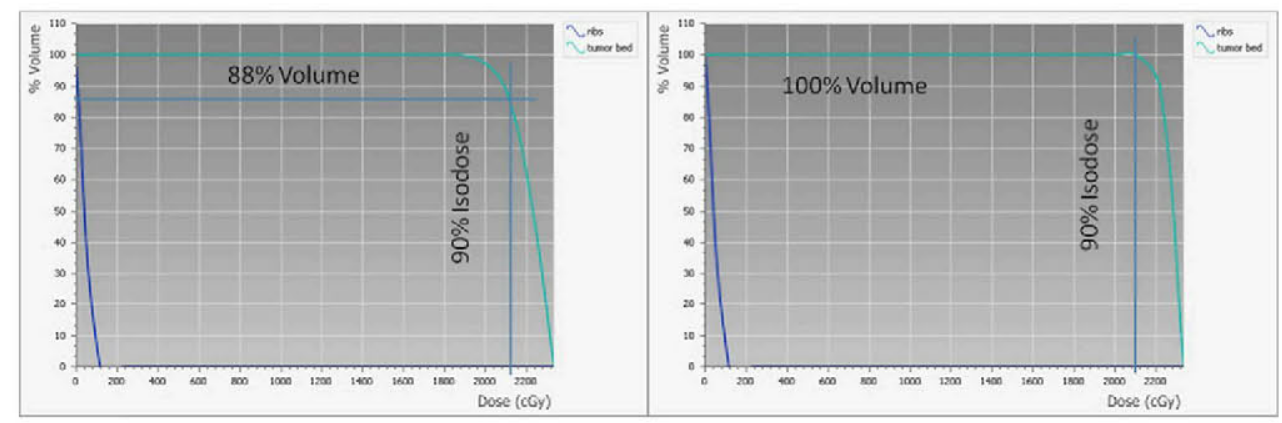

Figure 11. (Top) Representation of the $90 \%$ isodose curve for a $5 \mathrm{~cm}$ (top-left) and a $6 \mathrm{~cm}$ (top-right) applicator for case 1 . An arrow is used to highlight poor coverage on the sides of the tumour bed. (Bottom) Representation of the corresponding DVH, where the vertical line represents $90 \%$ of the prescribed dose. Isodoses curves are represented for the $95 \%$ (red) and $90 \%$ (orange) of the maximum dose.

caused by the artefacts by replacing pixel values with the expected materials. However, the correspondence between the simulated dose and the actual delivered dose is uncertain. The simulation required 6.2 million histories and took $18 \mathrm{~s}$ to meet a $2 \%$ level of uncertainty. This computation time is small enough to not interfere with the surgical procedure.

\section{Discussion and conclusions}

This work has analysed the conditions under which a custom implementation of an existing MC algorithm meets the requirements for interactive use in IOERT during planning and treatment. The availability of an MC method capable of satisfying time and accuracy constraints in all scenarios would pave the way for systematic use of treatment planning in the IOERT clinical routine. The computation of volumetric distributions is a clear step forward with respect to reporting the $90 \%$-dose curve and the maximum dose, which is currently the standard protocol (Beddar et al 2006).

As part of this work, the accuracy of the MC tool implemented to reproduce dose profiles in water and slab phantoms was assessed at different energies. These tests verified that the optimized algorithm was at least as accurate as the original implementation, even in the presence of sharp inhomogeneities, while requiring a fraction of the computation time. 


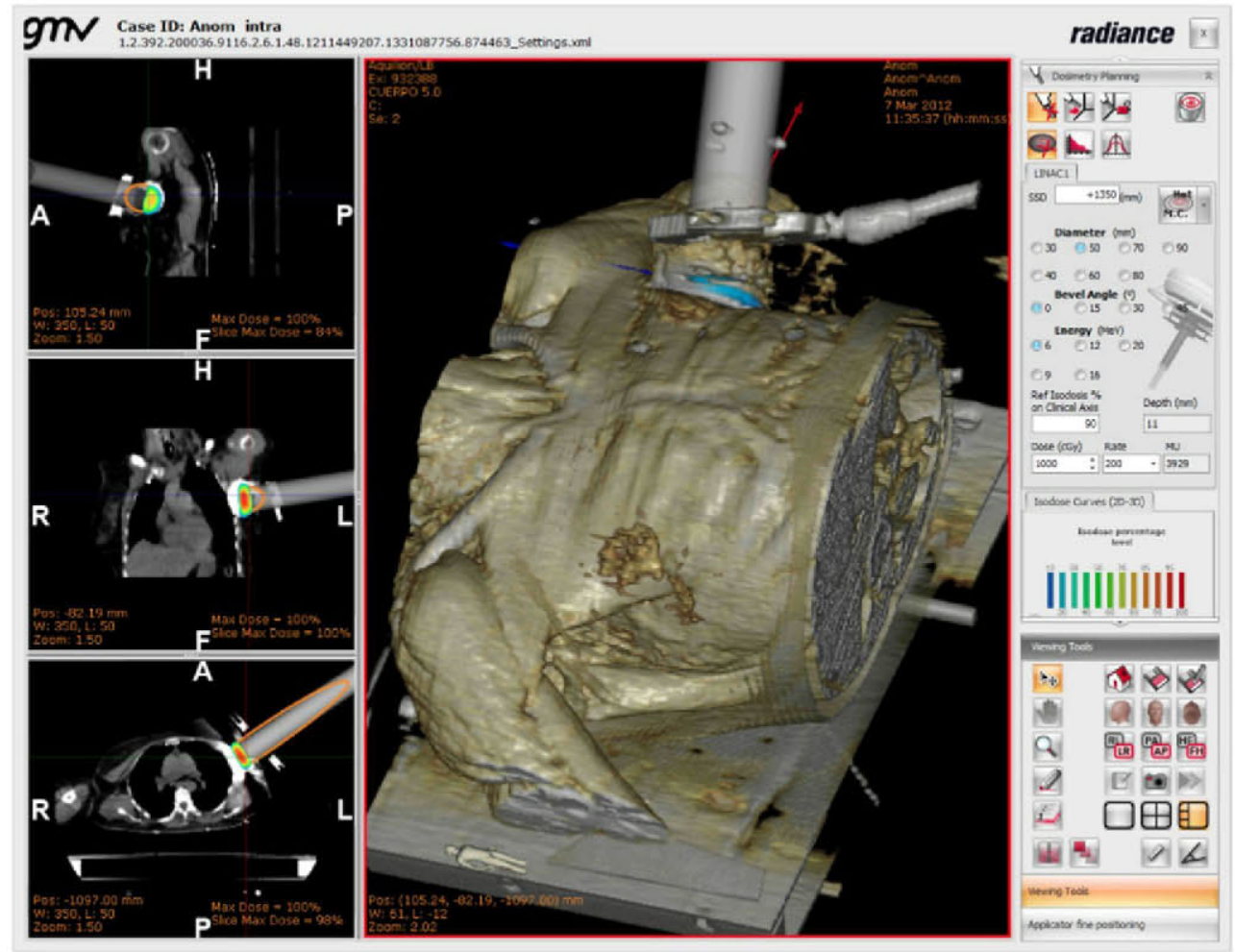

Figure 12. TPS interface showing the MC simulation output for an intraoperative treatment with a $6 \mathrm{MeV}$ electron beam and a $5 \mathrm{~cm}$ applicator (case 2). The breast is protected with a composite shield by a RO and the applicator is kept in place by a holder. The pixel size of the intraoperative CT image is $1 \times 1 \times 5 \mathrm{~mm}^{3}$.

To assess the level of interactivity that can be expected, execution times to meet a predefined uncertainty level were evaluated using a collection of PHSPs capable of reproducing experimental measurements in a water phantom for nominal energies in the range of $6-20 \mathrm{MeV}$. From these simulations, it was concluded that all set-ups commonly used in the clinical routine could be solved in less than $2.5 \mathrm{~min}$ if the voxel size was kept above $2 \times 2 \times 2 \mathrm{~mm}^{3}$.

The actual level of interactivity that can be achieved has been illustrated with two early breast cancer test cases for which treatment plans had been defined, considering a $6 \mathrm{MeV}$ beam energy and including protection around the lung. It is in this scenario where the use of a fast MC tool becomes more relevant; analytical methods, such as pencil beam, show serious limitations when inhomogeneous phantoms are evaluated because of high-density inhomogeneities and the use of a relatively low-energy electron beam (Ding et al 2005). Computation time is also a critical issue, particularly when the RO needs to simulate multiple alternatives or to confirm dose distributions for a given set-up in the OR. In both test case scenarios, the dose was computed in less than $35 \mathrm{~s}$ for an uncertainty lower than $2 \%$ of the dose at the maximum. These results confirm the interactive usability of this MC technique, without compromising accuracy in dose predictions, provided that the CT images used as input to the model are adequate for accurate dosimetry. Therefore, we may conclude that computation time or accuracy are no longer a bottleneck for the application of MC simulation in IOERT when used as an additional step for quality control and documentation of the procedure. 
From the clinical perspective, the validity and usefulness of TPS has been assessed in a comparative study conducted with the participation of three ROs (Calvo et al 2014). Fifteen cases based on the most representative locations of IOERT treatments were evaluated, with the conclusion that the TPS offers a new imaging expansion for IOERT, in the context of a multidisciplinary approach to optimize and define the treatment parameters. However, the actual use of the TPS in the clinic requires the consideration and discussion of other factors. With regard to the use of the TPS with preoperative CT images, which is the planning workflow for IOERT that was originally proposed (Pascau et al 2012), the matter of the level of accuracy that can be obtained using preoperative images to estimate the actual dose distribution is a topic that is still under investigation, beyond the preliminary results given by (Pascau et al 2011).

Concerning the intraoperative use of the TPS, it is intuitive that this is the best approach to fully understand the patient's actual anatomy during treatment and should be considered if accurate dose estimations are intended. To obtain updated patient imaging during the IOERT procedure, portable devices should be considered in the protocol (Siewerdsen 2001). Nevertheless, several challenges, which are still being studied, must be taken into account to acquire and post-process such images. These include the effects of shielding disks on the image, the image quality and potential dosimetric limitations of portable CT devices or the methodology to transfer segmented regions and volumes from the preoperative to intraoperative images, in order to avoid manual segmentation by the RO in the time-constrained intraplanning phase. In any case, the $\mathrm{MC}$ workflow proposed here could assist in evaluating the potential use of a portable CT scanner for dose estimations in IOERT. The benefits of intraoperative imaging in the clinical workflow should also be confirmed for different treatment locations.

In IOERT, it is common to use a shielding disk to protect the organs at risk, but this introduces important artefacts in the CT that may limit the use of intraplanning. Further study of the sensitivity of dose distributions to these artefacts is needed, as well as investigating alternative techniques to minimize or remove them. In fact, the use of this MC tool in the TPS could be a valuable tool to assess the benefits and effects of different protection materials.

Finally, to minimize the delay in the procedure due to intraplanning computations, segmentation contours from preoperative images could be transferred to the intraoperative study by means of non-rigid image registration techniques.

The proposed MC-optimized tool and the workflow presented narrow the gap between external and internal radiotherapy, providing the $\mathrm{RO}$ with mechanisms to estimate the actual dose delivered to the patient in a complex clinical scenario, including the effects of protection systems and bolus. The integration of the MC tool in a user-friendly TPS will help in the standardization of IOERT and increase its applicability. Additionally, these techniques will enable improved handling of patients, an improved analysis of the treatment outcome, as well as a dose summation in those cases where IOERT is combined with external radiotherapy as a boost strategy.

\section{Acknowledgments}

This work was supported by the Spanish Ministry of Science and Innovation, currently integrated in the Ministry of Economy, under grants IPT-300000-2010-003, IPT-20120401-300000, TEC2010-21619-C04, TEC2013-48251-C2, TEC2011-28972-C02-02 and FPA2010-17142, and by Comunidad de Madrid grant TOPUS P2013/MIT-3024.

We also acknowledge the European Union for their funding through FP7 project BiopsyPen and the European Fund for Regional Development (FEDER). 


\section{References}

Almond P R, Biggs P J, Coursey B M, Hanson W F, Huq M S, Nath R and Rogers D W O 1999 AAPM's TG-51 protocol for clinical reference dosimetry of high-energy photon and electron beams Med. Phys. 26 1847-70

Beddar A S, Biggs P J, Chang S, Ezzell G A, Faddegon B A, Hensley F W and Mills M D 2006 Intraoperative radiation therapy using mobile electron linear accelerators: report of AAPM Radiation Therapy Committee Task Group No. 72 Med. Phys. 33 1476-89

Bielajew A F and Wilderman S J 2000 Innovative electron transport methods in EGS5 Proc. of the Second Int. Workshop on EGS4 (Japan: KEK Proceedings 2000-20) pp 1-10

Björk P, Knöös T and Nilsson P 2004 Measurements of output factors with different detector types and Monte Carlo calculations of stopping-power ratios for degraded electron beams Phys. Med. Biol. 49 4493-506

Calvo F A, Murillo L A, Sallabanda M, Martinez-Villanueva J and Solea C V 2014 Imaging opportunities for treatment planning in intraoperative electron beam radiotherapy (IOERT): developments in the context of RADIANCE system Rep. Pract. Oncol. Radiother. $19239-45$

Catalano M, Agosteo S, Moretti R and Andreoli S 2007 Monte Carlo simulation code in optimisation of the IntraOperative Radiation Therapy treatment with mobile dedicated accelerator J. Phys.: Conf. Ser. 74012002

Chetty I et al 2007 Report of the AAPM Task Group No. 105: issues associated with clinical implementation of Monte Carlo-based photon and electron external beam treatment planning $\mathrm{Med}$. Phys. 34 4818-53

Cygler J, Daskalov G, Chan G and Ding G 2004 Evaluation of the first commercial Monte Carlo dose calculation engine for electron beam treatment planning Med. Phys. 31 142-53

Cygler J, Li X A, Ding G X and Lawrence E 1997 Practical approach to electron beam dosimetry at extended SSD Phys. Med. Biol. 421505

Dall'Oglio S 2013 'Study protocol: electrons for intraoperative radiotherapy in selected breast-cancer patients: late results of the Montpellier phase II trial' Radiat. Oncol. 8191 Radiation Oncology, comments to the manuscript

Ding G, Cygler J, Yu C, Kalach N and Daskalo V G 2005 A comparison of electron beam dose calculation accuracy between treatment planning systems using either a pencil beam or a Monte Carlo algorithm Int. J. Radiat. Oncol. Biol. Phys. 63 622-33

Fastner G et al 2013 IORT with electrons as boost strategy during breast conserving therapy in limited stage breast cancer: long term results of an ISIORT pooled analysis Radiother. Oncol. $108279-86$

García-Vázquez V, Marinetto E, Santos-Miranda J A, Calvo F A, Desco M and Pascau J 2013 Feasibility of integrating a multi-camera optical tracking system in intra-operative electron radiation therapy scenarios Phys. Med. Biol. 58 8769-82

Gunderson L L, Willett C G, Harrison L B and Calvo F A 2011 Intraoperative Irradiation: Techniques and Results (New York: Humana)

Herranz E 2013 Simulaciones Monte Carlo para Radioterapia Intraoperatoria con haces de electrones PhD Thesis Universidad Complutense, Madrid

Herranz E, Herraiz J L, Ibañez P, Perez-Liva M, Puebla R, Cal-Gozález J, Guerra P, Rodríguez R, Illana C and Udías J M 2013 Phase space determination of electron beam for intraoperative radiation therapy from measured dose data Phys. Med. Biol. Submitted

Hissoiny S and Ozell B 2011 GPUMCD: a new GPU-oriented Monte Carlo dose calculation platform Med. Phys. 38 754-65

Iaccarino G, Strigari L, D'Andrea M, Bellesi L, Felici G, Ciccotelli A, Benassi M and Soriani A 2011 Monte Carlo simulation of electron beams generated by a $12 \mathrm{MeV}$ dedicated mobile IORT accelerator Phys. Med. Biol. 56 4579-96

Jia X, Gu X, Graves Y J, Folkerts M and Jiang S B 2011 GPU-based fast Monte Carlo simulation for radiotherapy dose calculation Phys. Med. Biol. 56 7017-31

Kawrakow I and Bielajew A F 1998 On the condensed history technique for electron transport Nucl. Instrum. Methods Phys. Res. B 142 253-80

Kawrakow I and Rogers D W 2000 The EGSnrc code system: Monte Carlo simulation of electron and photon transport Technical Report PIRS-701 ed N R C o, Ottawa, Canada 
Kock E A D and Schreuder A N 1996 Letters to the editor. Comments 'The calibration of CT Hounsfield units for radiotherapy treatment planning' Phys. Med. Biol. 41 1524-7

L'Ecuyer P 1998 Tables of maximally equidistributed combined LFSR generators Math. Comput. 68 261-9

L'Ecuyer P and Hellekalek P 1998 Random number generators: selection criteria and testing Random and Quasi-Random Point Sets (New York: Springer) pp 223-65

L'Ecuyer P and Panneton F 2005 Fast random number generators based on linear recurrences modulo 2: overview and comparison Proc. of the Winter Simulation Conf. (Orlando, FL: IEEE) p 10

Lamanna E, Gallo A, Russo F, Brancaccio R, Soriani A and Strigari L 2012 Intra-operative radiotherapy with electron beam Modern Practices in Radiation Therapy ed G Natanasabapathi (Rijeka: InTech)

Low D A, Harms W B, Mutic S and Purdy J A 1998 A technique for the quantitative evaluation of dose distributions Med. Phys. 25 656-61

Palta J, Biggs P, Hazle J, Huq M, Dahl R, Ochran T, Soen J, Dobelbower R J and McCullough E 1995 Intraoperative electron beam radiation therapy: technique, dosimetry, and dose specification: report of task force 48 of the Radiation Therapy Committee, American Association of Physicists in Medicine Int. J. Radiat. Oncol. Biol. Phys. 33 725-46

Pascau J, Santos Miranda J, Calvo F, Bouché A, Morillo V, González-San Segundo C, Ferrer C, López Tarjuelo J and Desco M 2012 An innovative tool for intraoperative electron beam radiotherapy simulation and planning: description and initial evaluation by radiation oncologists Int. J. Radiat. Oncol. Biol. Phys. 83 e287-95

Pascau J, Santos-Miranda J, San-Segundo C G, Illana C, Valdivieso M, García-Vazquez V, Marinetto E, Calvo F and Desco M 2011 Intraoperative Imaging in IOERT sarcoma treatment: initial experience in two clinical cases Int. J. Radiat. Oncol. Biol. Phys. $81 \mathrm{~S} 90$

Righi S, Karaj E, Felici G and Di Martino F 2013 Dosimetric characteristics of electron beams produced by two mobile accelerators, Novac7 and Liac, for IOERT through Monte Carlo simulation J. Appl. Clin. Med. Phys. 143678

Rogers D W O and Mohan R 2000 Questions for comparison of clinical Monte Carlo codes 13th Int. Conf. the Use of Computers in Radiotherapy ed W Schlegel and T Bortfeld (Heidelberg: Springer) pp $120-2$

Rosi A and Viti V 2004 Guidelines for quality assurance in IOERT Oncología 27 62-95

Russo G, Casarino C, Arnetta G, Candiano G, Stefano A, Alongi F, Borasi G, Messa C and Gilardi M C 2012 Dose distribution changes with shielding disc misalignments and wrong orientations in breast IOERT: a Monte Carlo_GEANT4 and experimental study J. Appl. Clin. Med. Phys. 133817

Schneider U, Pedroni E and Lomax A 1996 The calibration of CT Hounsfield units for radiotherapy treatment planning Phys. Med. Biol. 41 111-24

Sempau J, Wilderman S J and Bielajew A F 2000 DPM, a fast, accurate Monte Carlo code optimized for photon and electron radiotherapy treatment planning dose calculations Phys. Med. Biol. $452263-91$

Siewerdsen J H 2001 Cone-beam CT with a flat-panel detector: from image science to image-guided surgery Nucl. Instrum. Methods Phys. Res. A 648 S241-50

Vanderstraeten B et al 2007 Conversion of CT numbers into tissue parameters for Monte Carlo dose calculations: a multi-centre study Phys. Med. Biol. 52 539-62

Walters B R B, Kawrakow I and Rogers D W O 2002 History by history statistical estimators in the BEAM code system Med. Phys. 29 2745-52 